\title{
The association between early-life salivary cytokines in premature infants and asthma development
}

\author{
Ying Lun $\mathrm{Hsu}^{1}$, Hsin-Chun Huang ${ }^{1}$, Ting-Yu Su${ }^{1}$, Chih-Hsing Hung ${ }^{2}$, and I-Lun Chen ${ }^{1}$ \\ ${ }^{1}$ Chang Gung Memorial Hospital Kaohsiung Branch \\ ${ }^{2}$ Kaohsiung Medical University Hospital
}

June 5, 2020

\begin{abstract}
Background Prematurity is a well-known risk factor for asthma. Biomarkers from sputum or serum were determined to have association with childhood asthma but no existing study has focused on salivary cytokines nor on prematurity. Thus, our study aimed to determine the relationship between childhood asthma and salivary cytokines in the early life of prematurity. Methods Preterm neonates from August 2012 to May 2017 were recruited and those with bacterial infection within seven days of life, maternal sepsis, and maternal clinical chorioamnionitis were excluded. Their gestational age, birth body weight, comorbidities, intubation duration, and infectious times during hospitalization were documented. The salivary cytokines on the first (D1) and seventh (D7) day of life were detected by MILLPLEX@ MAP Human multiplex assay. They were followed up and divided into asthma and non-asthma groups. Kaplan-Meier or Cox-regression were used for analyzing the data. Results A total of 125 preterm infants were enrolled with 21 in the asthma group and 104 in the non-asthma group. Patients in the asthma group had longer duration of intubation $(p<0.001)$ and higher re-hospitalization rate for respiratory tract infection $(p<0.001)$, especially for respiratory syncytial virus bronchiolitis $(p=0.008)$. For salivary cytokines, the levels of D1 interleukin (IL)-8 were significantly higher in the asthma group $(p=0.04)$. Conclusions Salivary IL- 8 at one day of life was associated with the development of asthma in childhood of premature neonates. Therefore, we can intervene early on these high-risk premature infants to prevent the occurrence of asthma.
\end{abstract}

The association between early-life salivary cytokines in premature infants and asthma development

Ying-Lun Hsu, MD ${ }^{1}$, Hsin-Chun Huang, MD., PhD ${ }^{1,2}$, Ting-Yu Su, MD ${ }^{1}$, Chih-Hsing Hung, MD., PhD ${ }^{3,4,5,6}$, I-Lun Chen, MD., $\mathrm{PhD}^{1}$

${ }^{1}$ Department of Pediatrics, Kaohsiung Chang Gung Memorial Hospital, Kaohsiung, Taiwan.

${ }^{2}$ School of Traditional Chinese Medicine, College of Medicine, Chang Gung University, Linkou, Taiwan

${ }^{3}$ Graduate Institute of Clinical Medicine, College of Medicine, Kaohsiung Medical University, Kaohsiung, Taiwan

${ }^{4}$ Department of Pediatrics, Kaohsiung Medical University Hospital, Kaohsiung Medical University, Kaohsiung, Taiwan;

${ }^{5}$ Department of Pediatrics, Kaohsiung Municipal Hsiao-Kang Hospital, Kaohsiung, Taiwan; Department of Pediatrics, Faculty of Medicine, College of Medicine, Kaohsiung Medical University, Kaohsiung, Taiwan;

${ }^{6}$ Research Center for Environmental Medicine, Kaohsiung Medical University, Kaohsiung, Taiwan.

Email: 
Ying-Lun Hsu: alenalenhsu@gmail.com

Hsin-Chun Huang: hhuang@cgmh.org.tw

Ting-Yu Su: drteneesu@gmail.com

Chih-Hsing Hung: pedhung@gmail.com

Corresponding author:

I-Lun Chen, MD., PhD

Department of Pediatrics

Kaohsiung Chang Gung Memorial Hospital

123, Dapi Rd. Niaosong Dist, Kaohsiung City 83301 Taiwan

Fax: 886-7-7338009; Tel: 886-7-7317123

E-mail: memeo@cgmh.org.tw

Keywords: Asthma and early wheeze; interleukin-8; prematurity; respiratory syncytial virus.

Running title: Asthma and salivary cytokines in prematurity

\section{Abstract}

\section{Background}

Prematurity is a well-known risk factor for asthma. Biomarkers from sputum or serum were determined to have association with childhood asthma but no existing study has focused on salivary cytokines nor on prematurity. Thus, our study aimed to determine the relationship between childhood asthma and salivary cytokines in the early life of prematurity.

\section{Methods}

Preterm neonates from August 2012 to May 2017 were recruited and those with bacterial infection within seven days of life, maternal sepsis, and maternal clinical chorioamnionitis were excluded. Their gestational age, birth body weight, comorbidities, intubation duration, and infectious times during hospitalization were documented. The salivary cytokines on the first (D1) and seventh (D7) day of life were detected by MILLPLEX@ MAP Human multiplex assay. They were followed up and divided into asthma and non-asthma groups. Kaplan-Meier or Cox-regression were used for analyzing the data.

\section{Results}

A total of 125 preterm infants were enrolled with 21 in the asthma group and 104 in the non-asthma group. Patients in the asthma group had longer duration of intubation $(p<0.001)$ and higher re-hospitalization rate for respiratory tract infection $(p<0.001)$, especially for respiratory syncytial virus bronchiolitis $(p=$ 0.008). For salivary cytokines, the levels of D1 interleukin (IL)-8 were significantly higher in the asthma group $(p=0.04)$.

\section{Conclusions}

Salivary IL-8 at one day of life was associated with the development of asthma in childhood of premature neonates. Therefore, we can intervene early on these high-risk premature infants to prevent the occurrence of asthma.

\section{Introduction}

Asthma is a chronic airway disease that causes recurrent symptoms such as wheezing, coughing, shortness of breath, and chest tightness. Asthma affected about $15 \%$ of school-age children in Taiwan, and the prevalence among school-age children was increasing in recent years ${ }^{1}$. Children who were born prematurely were known 
to have a higher risk of asthma ${ }^{2,3}$; however, the pathophysiology of asthma in former preterm children may be different from term children. Fewer gestational age (GA), lower birth body weight (BBW) and greater infant weight gain are presumed risk factors of asthma in preterm $\mathrm{birth}^{3,4}$. The pre-existing genetic and environmental factors result in premature delivery, the immature lung development and immune system with susceptibility to recurrent pulmonary infection, and postnatal lung injuries from barotrauma of ventilator and oxygen toxicity may impair these premature infants' lung function ${ }^{5,6}$. Some evidence has implied that preterm birth would develop respiratory disease in later life, especially obstructive lung disease with impaired lung function, leading to higher asthma and chronic obstructive pulmonary disease prevalence compared to term birth ${ }^{4,5}$. Recent advances in neonatal intensive care have greatly increased the survival rate of extremely preterm infants.

Asthma is an inflammatory disease and the airway wall is infiltrated by eosinophils, T lymphocytes, macrophages/monocytes, and mast cells, and sometimes by neutrophils in the chronic airway inflammation ${ }^{7,8}$. Cytokines especially interleukins derived from T-helper-2 (Th2) cells, such as interleukin (IL)-4, IL-5, and IL-13, were likely to play an important role in the inflammatory process of asthma ${ }^{9,10}$. However, some studies showed that asthmatic children who were born at preterm may have a lower incidence of atopy ${ }^{11}$. The result indicates that the cause of asthma in former prematurity may be different from asthma in former term babies. Up to date, there is no study focuses on the relationship between asthma and salivary cytokines of preterm. Thus, the aim of this study was to use a non-invasive way such as salivary cytokines and a risk factor to predict asthma in the early life of premature infants.

\section{Method}

\section{Study population}

Premature babies who were born at or less than gestational age 34 weeks from August 2012 to May 2017 were enrolled in this study after signing the informed consent by their parents or guardians. Besides the patients who were born at our hospital, those who were out-born but admitted to our neonatal intensive care unit were also included. In addition, we only enrolled patients who needed respiratory support after birth, such as nasal continues positive airway pressure, nasal intermittent mandatory ventilator or mechanical ventilator. We excluded patients with bacterial infection within seven days of life, maternal sepsis and maternal clinical chorioamnionitis since these factors may influence early-life cytokines levels. Babies with bacterial infection were diagnosed by clinical symptoms and/or proven culture from bio-fluid, such as blood, urine, or cerebral spinal fluid. The clinical chorioamnionitis was defined as maternal fever, uterine fundal tenderness and purulent, or foul amniotic fluid and bacterial growth in amniotic fluid culture or placenta. This study was approved by the Institutional Review Board of Chang Gung Memorial Hospital.

The admitted history of patients as GA, BBW, Apgar score, the duration of intubation, the frequencies of infectious episodes and comorbidities of premature infants, such as bronchopulmonary dysplasia (BPD), significant patent ductus arteriosus (sPDA), respiratory distress syndrome (RDS), periventricular leukomalacia (PVL), intraventricular hemorrhage (IVH), retinopathy of prematurity (ROP) and necrotizing enterocolitis (NEC) were documented. The information regarding maternal history such as colonization of group B streptococcus (GBS), use of prenatal steroid, smoking history during pregnancy, maternal disease as preeclampsia, fever, gestational diabetes (GDM), and hours of premature rupture of membrane (PROM) were also recorded.

The definition of bronchopulmonary dysplasia is that patients require more than $21 \%$ oxygen for 28 days after birth $^{12,13}$. A significant PDA means the diameter of PDA is larger than $2.5 \mathrm{~mm}^{14}$. IVH grading was based on Papile grading system ${ }^{15}$, NEC staging was defined according to the modified Bell staging ${ }^{16}$, and ROP stage was classified based on The international classification of retinopathy of prematurity (ICROP) ${ }^{17}$.

\section{Saliva collection and cytokines detection}

The saliva was collected on the first (D1) and seventh (D7) day of life. In order to avoid independent diurnal rhythms of salivary cytokines, the sample was collected at seven o'clock in the morning before the neonates 
were fed ${ }^{18}$. The baby was put in a supine position with head at the midline, a 6 or 8 Fr suction tube was inserted into the baby's mouth to obtain at least $0.5 \mathrm{~mL}$ saliva in a sterile tube. The sample was immediately sent to the laboratory and stored at $-80^{\circ} \mathrm{C}$ until batch analysis ${ }^{19}$.

The salivary cytokines levels, included interferon (IFN)- $\alpha 2$, IFN- $\gamma$, interleukin (IL)-6, IL-8, IL-10, IL-17, IL33 , and tumor necrosis factor (TNF)- $\alpha$, were analyzed by multiplexed fluorescent bead-based immunoassays, in accordance with the manufacturer's instruction. In brief, $25 \mu \mathrm{L}$ of saliva supernatant was added to antihuman multi-cytokine magnetic beads (EMD Millipore, Billerica, MA, USA). Quality controls were included to ensure the data was reliable. Detection was then performed using the Luminex ${ }^{\circledR}$ MAGPIX ${ }^{\circledR}$ Multiplexing instrument with xPonent ${ }^{\circledR} 4.2$ software and data analysis was employed with MILLIPLEX ${ }^{\circledR}$ Analyst 5.1 software $^{19}$. Calibration of cytokine concentrations were determined by interpolation of a series of well-known standard samples following the manufacturer's recommendation.

\section{Follow-up}

The qualified patients were divided into asthma and non-asthma groups. Our operational definition of asthma was either diagnosed by a pediatrician or using asthma medications, such as inhaled selective $\beta 2$ agonists and/or inhaled corticosteroid (ICS) treatments, more than twice in a year for at least one year and/or taking oral leukotriene modifiers for more than one month, and the patients were above 2 years old ${ }^{20}$. Neonatologists followed-up these patients at our out-patient department (OPD) and/or by phone interview to determine their status, respiratory symptoms, medication history and any ongoing or chronic illness. The follow-up time was calculated as the number of days from their birthday to the date of asthma diagnosis and to the date of their last OPD visiting or phone interview for the non-asthma group. In addition, the frequency of re-admission and the diagnosis of re-admission were documented from patients' medical records. The doses of palivizumab for respiratory syncytial virus (RSV) prevention and those who did not receive palivizumab injection were also collected. The usage of palivizumab given in this study was as recommendation of the Taiwan National Health Insurance (TNHI) policy (version before 2015.04.01). According to the policy, all premature infants with GA at or less than 28 weeks or at or less than 35 weeks with BPD would receive prophylaxis palivizumab $750 \mathrm{mg} / \mathrm{kg}$ intramuscularly every month for a total of six months. The first dose was given within one week before their discharging home.

\section{Statistical analysis}

The Cox regression and Kaplan-Meier test were used to analyze continuous and categorical variables, respectively. The demographic information and risk factors, such as GA, BBW, comorbidities of premature infants (BPD, RDS, sPDA, PVL, IVH, ROP and NEC), the frequency of re-admission due to respiratory infection, RSV infection and the doses of palivizumab injection, were compared between asthma and nonasthma groups. A significant level was set at $p<0.05$. All statistical analyses were performed by the IBM SPSS Statistics 25.0 software.

\section{Result}

A total of 186 preterm infants were recruited in this study at the beginning. Eighteen neonates died during their first hospitalization. Thirty-six of survivors were excluded due to maternal infection (33 neonates had maternal clinical chorioamnionitis, two had maternal sepsis, and one had Cytomegalovirus infection). Seven babies were excluded because of the bacterial infection within seven days of life (three had GBS infection, two had pneumonia, and two had sepsis). As the result, there were 125 infants recruited in this study.

These 125 infants were divided into asthma $(n=21)$ and non-asthma $(n=104)$ groups based on our operational definition of asthma. Table 1 shows the characteristics for the two groups. The mean of GA and BBW were 28.5 weeks, $1179.3 \mathrm{~g}$ and 29.5 weeks, $1272.2 \mathrm{~g}$ in asthma and non-asthma groups, respectively. Despite fewer gestational age and lower birth body weight noted in the asthma group, they did not reach statistical difference. There was also no significant difference in gender, mode of delivery, Apgar score at first and fifth minute between the two groups. Neither maternal status as GBS colonization, prenatal steroid use, smoking history during pregnancy, preeclampsia, maternal fever, gestational diabetes nor hours of 
premature rupture of membranes has significant difference between the two groups. Common comorbidities of prematurity, BPD, grade of RDS, frequency of infectious episodes, incidences of sPDA and PVL, the stage of IVH and NEC were not significantly different between the 2 groups neither. However, a significantly longer intubation period was found in the asthma group than the non-asthma group with $p<0.001$.

These 125 patients' atopic history, and their family atopic history were not significantly different in the two groups (Table 2). However, there was a significantly higher respiratory tract infection re-hospitalization frequencies in the asthma group $(p<0.001)$, and patients with RSV infection history were also likely to develop asthma, $p=0.008$. Six infants were diagnosed with laboratory-confirmed RSV infection and three of them developed asthma later. All of them have RSV infection before the asthma diagnosis. Nevertheless, the doses of Palivizumab did not show a significant difference between the two groups, $p=0.271$ (Table 3).

As for salivary cytokine study, the level of IFN- $\alpha 2$, IFN- $\gamma$, IL-6, IL-8, IL-10, IL-17, IL-33, TNF- $\alpha$ on the first day (D1) and seventh day (D7) of life were analyzed. The result showed D1 IL-8 level was significantly higher in the asthma group, $p=0.04$, Hazard ratio 1.000014, with 95\% CI 1.000001 1.000028, (Table 4 and Table 5). The result suggested the 10000 pg IL-8 increase in saliva on the first day of life is associated with a $14 \%$ increase in developing asthma later in their childhood.

\section{Discussion}

The result of this study showed that IL-8 level in saliva at one day of life was higher in the asthma group. Besides, longer intubation periods and higher respiratory tract infection frequencies, especially RSV infection, were also associated with asthma development. In addition, the atopy incidence, family history of atopy and maternal smoking history were not significantly higher in the asthma group. This result was different from the previous study of asthma in term birth children ${ }^{21}$.

To date, there was no similar study focused on the association between cytokines in prematurity and asthma, neither in serum nor in saliva. Therefore, in addition to show the difference of asthma characteristic in preterm birth than term birth, our study is the first study to determine the relationship of salivary cytokine in prematurity and asthma.

IL-8 is a neutrophil-activating cytokine, also known as a strong indicator of inflammation, in both acute or chronic inflammation. When exposed to stimulus like IL-1 or TNF, IL-8 is produced by phagocytes and mesenchymal cells and then activates neutrophils by recruiting and activating neutrophils from peripheral

blood into tissue ${ }^{22,23}$. Elevated IL-8 level is observed in many inflammatory diseases such as pediatric acute respiratory distress syndrome, acute respiratory failure ${ }^{24}$, and asthma ${ }^{25,26}$. In our study, D1 IL-8 level in saliva was significantly higher in the asthma group. Previous evidence has shown that elevated IL-8 in amniotic fluid was associated with preterm premature of membranes, chorioamnionitis and preterm birth ${ }^{27}$. This implied that the elevated D1 IL-8 level in saliva of these premature infants in the asthma group may concur with stress, infection or inflammation in the fetal stage, which resulted in asthma developing in their later life. Furthermore, there was a moderate negative-correlation between GA and IL-8 level $(p<0.001, r$ $=0.51$, Figure 1A), which means patients with fewer gestational age had higher IL-8 level at one day old. The fewer GA is also a known risk factor of asthma developing in prematurity ${ }^{3}$. Therefore, the fewer GA might result from elevated IL-8 level and contributed to the asthma development in childhood.

RSV is the most common pathogen in infantile bronchiolitis, and many studies have demonstrated its link to develop asthma ${ }^{28,29}$. It is still in a debate that some have claimed lower respiratory tract infections such as RSV infection in early life may cause asthma development ${ }^{30}$, while some have assumed that RSV infection only identifies infants who genetically tend to develop wheezing and subsequent asthma since there may be a common genetic background of these two diseases ${ }^{31}$. In addition, some studies have demonstrated that gene polymorphisms of IL- 8 could increase disease severity in infants of RSV infection ${ }^{32,33}$, which might be a predisposing factor to develop asthma after RSV bronchiolitis. Thus, the gene predisposition with higher IL-8 level of these premature infants or elevated IL- 8 secretion in airway induced by concurrent RSV infection may lead to a high prevalence of asthma in childhood. 
Another result worth noting in our study is that the doses of palivizumab between the asthma and the nonasthma group were not significantly different. Palivizumab is the FDA-approved monoclonal antibody which is directed against the RSV fusion protein ( $\mathrm{F}$ glycoprotein) and is recommended as prophylaxis for infants who were younger than 1-year-old with high-risk by the American Academy of Pediatrics ${ }^{34}$. The dose of palivizumab given in this study was based on the recommendation from the TNHI policy. A total of six infants had RSV-infection, one of them did not receive palivizumab due to not conform to the TNHI policy, one infant had RSV infection during palivizumab prophylaxis period, and the other 4 infants had RSV infection not within palivizumab prophylaxis period. Three of these six patients had RSV infection and developed asthma in their childhood, one got RSV infection before receiving palivizumab prophylaxis, and the other got RSV infection after completed 6 doses of palivizumab prophylaxis (figure 1B). Although Palivizumab has been proved to reduce hospitalizations of severe RSV infection ${ }^{35}$ and has an immunomodulation effect ${ }^{36}$. However, it seems that palivizumab would not prevent asthma development as shown in this study. This was in consistent with other study ${ }^{37}$.

In our study, some known risk factors ${ }^{21}$ of asthma such as fewer gestational age, lower birth weight, and cesarean section did not have a significant difference between the two groups. Though not significant, the asthma group in our study did have a fewer gestational age $(28.5$ vs. 29.5 weeks, $p=0.068)$ and lower birth weight (1186.4 vs. $1270.7 \mathrm{~g}, p=0.316)$. The probable reasons that these known risk factors did not reach statistical significance may relate to our inclusion criteria. We only included prematurity at or fewer than gestational age of 34 weeks with ventilator support, which may lead to the selection bias.

Upon family atopic history and the patients' atopic history, the results showed no significant difference in the two groups. However, a previous study had revealed that the incidence of atopy was lower in children who were born preterm than those born at term according to skin prick test, but the incidence of asthma was not decreased ${ }^{11}$. In addition, another study showed that preterm birth was associated with increased risk for severe asthma and decreased risk for severe atopic dermatitis ${ }^{38}$. Thus, the mechanism of asthma development in previous preterm birth may be different from term birth infants.

It is worth noted that the asthma group had significantly longer intubation duration $(p<0.001)$. Previous studies have demonstrated the association between mechanical ventilation with $\mathrm{BPD}^{39}$, but no study has mentioned about the development of asthma. The mechanism of asthma in previous prematurity may cause by multiple factors such as ventilator induce injury to the immature lungs and recurrent virus infection. Besides, the longer intubation duration has a strong negative correlation with fewer gestational age $(p<$ $0.001, r=0.65$, figure 1C), which means patients with fewer gestational age had longer intubation period. Younger gestational age is a known risk factor of asthma ${ }^{3}$ though not significant in our study. Thus, their immature lung or lung injury resulted from prolonged ventilator used might tend to develop asthma in later life.

Asthma is an inflammatory disease and cytokines were known to play a role in promoting and mediating the inflammatory process. Therefore, knowing the correlation between cytokines, such as salivary IL-8 in our study, and asthma could help us to have early intervention on the high-risk patients with strategies like extubating as soon as possible, encouraging feeding with breast milk or hydrolyzed formula ${ }^{40}$ and avoiding allergen contacts to prevent asthma development. Besides, since RSV infection has a positive correlation with asthma, to extend RSV monoclonal antibody, palivizumab, injection may be considered for these high IL-8 level infants.

The diagnosis of asthma in children under 5 years was difficult since it is hard to perform a lung function test as spirometry on children under 5 years old. Therefore, many pediatric patients were diagnosed of asthma by history with typical asthma symptoms such as recurrent wheezing and cough, which vary over time and in intensity. It often occurs or worsen at night and may trigger by exercise, cold air and allergens, and empirical treatment response with ICS or bronchodilators. The asthma definition in this study was based on the clinical diagnosis and medication records.

Furthermore, we know that the environmental factors have an important impact on asthma development ${ }^{21}$. 
However, it is nearly impossible for us to eliminate the environmental factor in our study. Even if these patients were all living in southern Taiwan, the air pollution condition would have a large range of differences.

\section{Conclusion}

The elevated level of salivary IL- 8 in the early life of prematurity has a positive association with asthma development in their childhood. The result would alert clinical practitioner to early intervene the high-risk patients to prevent asthma occurrence.

\section{Conflict of interest:}

The authors have no conflict of interests to declare

\section{Funding statement}

This study was supported by grant CMRPG8F0711 (I-Lun Chen), and CMRPG8E0971 (Hsin-Chun Huang) from Kaohsiung Chang Gung Memorial Hospital.

\section{Acknowledgments:}

1. We thank our colleagues of stem cell research core laboratory who provided insight and expertise to assist the research.

2. We appreciated the Biostatistics Center, Kaohsiung Chang Gung Memorial Hospital for statistic consultation.

\section{Reference}

1. Chen BY, Chen CH, Chuang YC, Wu YH, Pan SC, Guo YL. Changes in the relationship between childhood asthma and ambient air pollution in Taiwan: Results from a nationwide survey repeated 5 years apart.Pediatr Allergy Immunol. 2019;30(2):188-194.

2. Jaakkola JJ, Ahmed P, Ieromnimon A, et al. Preterm delivery and asthma: a systematic review and meta-analysis. J Allergy Clin Immunol. 2006;118(4):823-830.

3. Agnes M. M. Sonnenschein-van der Voort P. Preterm birth, infant weight gain, and childhood asthma risk: A meta-analysis of 147,000 European children. J Allergy Clin Immunol. 2014;133(5):1317-1329.

4. den Dekker HT, Sonnenschein-van der Voort AMM, de Jongste JC, et al. Early growth characteristics and the risk of reduced lung function and asthma: A meta-analysis of 25,000 children. J Allergy Clin Immunol. 2016;137(4):1026-1035.

5. Thunqvist P, Tufvesson E, Bjermer L, et al. Lung function after extremely preterm birth-A populationbased cohort study (EXPRESS).Pediatr Pulmonol. 2018;53(1):64-72.

6. Hwang JS, Rehan VK. Recent Advances in Bronchopulmonary Dysplasia: Pathophysiology, Prevention, and Treatment. Lung.2018;196(2):129-138.

7. Azzawi M, Bradley B, Jeffery PK, et al. Identification of activated T lymphocytes and eosinophils in bronchial biopsies in stable atopic asthma. Am Rev Respir Dis. 1990;142(6 Pt 1):1407-1413.

8. Fahy JV, Kim KW, Liu J, Boushey HA. Prominent neutrophilic inflammation in sputum from subjects with asthma exacerbation. J Allergy Clin Immunol. 1995;95(4):843-852.

9. Chung KF. Targeting the interleukin pathway in the treatment of asthma. The Lancet. 2015;386(9998):1086-1096.

10. Vizmanos-Lamotte G, Moreno-Galdo A, Munoz X, Gomez-Olles S, Gartner S, Cruz MJ. Induced sputum cell count and cytokine profile in atopic and non-atopic children with asthma. Pediatr Pulmonol.2013;48(11):1062-1069. 
11. Siltanen M, Wehkalampi K, Hovi P, et al. Preterm birth reduces the incidence of atopy in adulthood. $J$ Allergy Clin Immunol.2011;127(4):935-942.

12. Jobe AH, Bancalari E. Bronchopulmonary dysplasia. Am J Respir Crit Care Med. 2001;163(7):17231729 .

13. Day CL, Ryan RM. Bronchopulmonary dysplasia: new becomes old again!Pediatr Res. 2017;81(1-2):210213.

14. Cambier PA, Kirby WC, Wortham DC, Moore JW. Percutaneous closure of the small (less than 2.5 mm) patent ductus arteriosus using coil embolization. Am J Cardiol. 1992;69(8):815-816.

15. Papile LA, Burstein J, Burstein R, Koffler H. Incidence and evolution of subependymal and intraventricular hemorrhage: a study of infants with birth weights less than 1,500 gm. J Pediatr.1978;92(4):529-534.

16. Mupsilonller MJ, Paul T, Seeliger S. Necrotizing enterocolitis in premature infants and newborns. $J$ Neonatal Perinatal Med.2016;9(3):233-242.

17. The International Classification of Retinopathy of Prematurity revisited. Arch Ophthalmol. 2005;123(7):991-999.

18. Chiappelli F, Iribarren FJ, Prolo P. Salivary biomarkers in psychobiological medicine. Bioinformation. 2006;1(8):331-334.

19. Chen IL, Huang H-C, Ou-Yang M-C, Chen F-S, Chung M-Y, Chen C-C. A novel method to detect bacterial infection in premature infants: Using a combination of inflammatory markers in blood and saliva. J Microbiol Immunol Infect. 2019. DOI.org/10.1016/j.jmii.2019.11.002

20. Ducharme FM, Dell SD, Radhakrishnan D, et al. Diagnosis and management of asthma in preschoolers: A Canadian Thoracic Society and Canadian Paediatric Society position paper. Can Respir J.2015;22(3):135143.

21. Castro-Rodriguez JA, Forno E, Rodriguez-Martinez CE, Celedon JC. Risk and Protective Factors for Childhood Asthma: What Is the Evidence? J Allergy Clin Immunol Pract. 2016;4(6):1111-1122.

22. Baggiolini M, Walz A, Kunkel SL. Neutrophil-activating peptide-1/interleukin 8, a novel cytokine that activates neutrophils.J Clin Invest. 1989;84(4):1045-1049.

23. Marco Baggiolini, Clark-Lcwisb I. Interleukin-8, a chemotactic and inflammatory cytokine. FEBS Lett. 1992;307(1):97-101.

24. Flori H, Sapru A, Quasney MW, et al. A prospective investigation of interleukin-8 levels in pediatric acute respiratory failure and acute respiratory distress syndrome. Crit Care. 2019;23(1):128-128.

25. Norzila MZ, Fakes K, Henry RL, Simpson J, PG G. Interleukin-8 Secretion and Neutrophil Recruitment Accompanies Induced Sputum Eosinophil Activation in Children with Acute Asthma. Am J Respir Crit Care Med. 2000;161(3):769-747.

26. Hosoki K, Ying S, Corrigan C, et al. Analysis of a Panel of 48 Cytokines in BAL Fluids Specifically Identifies IL-8 Levels as the Only Cytokine that Distinguishes Controlled Asthma from Uncontrolled Asthma, and Correlates Inversely with FEV1. PLoS One.2015;10(5):e0126035.

27. Jia X. Value of amniotic fluid IL-8 and Annexin A2 in prediction of preterm delivery in preterm labor and preterm premature rupture of membranes. J Reprod Med. 2014;59(3-4):154-160.

28. Sigurs N, Aljassim F, Kjellman B, et al. Asthma and allergy patterns over 18 years after severe RSV bronchiolitis in the first year of life. Thorax. 2010;65(12):1045-1052. 
29. Korsten K, Blanken MO, Buiteman BJM, et al. RSV hospitalization in infancy increases the risk of current wheeze at age 6 in late preterm born children without atopic predisposition. Eur $J$ Pediatr.2019;178(4):455-462.

30. Wu P, Dupont WD, Griffin MR, et al. Evidence of a causal role of winter virus infection during infancy in early childhood asthma.Am J Respir Crit Care Med. 2008;178(11):1123-1129.

31. Young S, O'Keeffe PT, Arnott J, Landau LI. Lung function, airway responsiveness, and respiratory symptoms before and after bronchiolitis. Arch Dis Child. 1995;72(1):16-24.

32. J Hull, A Thomson, Kwiatkowski D. Association of respiratory syncytial virus bronchiolitis with the interleukin 8 gene region in UK families. Thorax. 2000;55(12):1023-1027.

33. Jeremy Hull, Hans Ackerman, Kate Isles, et al. Unusual Haplotypic Structure of IL8, a Susceptibility Locus for a Common Respiratory Virus.Am J Hum Genet. 2001;69(2):413-419.

34. Modified Recommendations for Use of Palivizumab for Prevention of Respiratory Syncytial Virus Infections. Pediatrics.2009;124(6):1694-1701.

35. Palivizumab, a humanized respiratory syncytial virus monoclonal antibody, reduces hospitalization from respiratory syncytial virus infection in high-risk infants. The IMpact-RSV Study Group.Pediatrics. 1998;102(3 Pt 1):531-537.

36. Bar-Yoseph R, Haddad J, Hanna M, et al. Long term follow-up of Palivizumab administration in children born at 29-32 weeks of gestation.Respir Med. 2019;150:149-153.

37. Scheltema NM, Nibbelke EE, Pouw J, et al. Respiratory syncytial virus prevention and asthma in healthy preterm infants: a randomised controlled trial. Lancet Respir Med. 2018;6(4):257-264.

38. Tronnes H, Wilcox AJ, Lie RT, Markestad T, Moster D. The association of preterm birth with severe asthma and atopic dermatitis: a national cohort study. Pediatr Allergy Immunol. 2013;24(8):782-787.

39. Jobe AH. Mechanisms of Lung Injury and Bronchopulmonary Dysplasia.Am J Perinatol. 2016;33(11):1076-1078.

40. Greer FR, Sicherer SH, Burks AW. The Effects of Early Nutritional Interventions on the Development of Atopic Disease in Infants and Children: The Role of Maternal Dietary Restriction, Breastfeeding, Hydrolyzed Formulas, and Timing of Introduction of Allergenic Complementary Foods. Pediatrics. 2019;143(4).

\section{Hosted file}

table 1.docx available at https://authorea.com/users/330315/articles/457134-the-associationbetween-early-life-salivary-cytokines-in-premature-infants-and-asthma-development

\section{Hosted file}

table 2.docx available at https://authorea.com/users/330315/articles/457134-the-associationbetween-early-life-salivary-cytokines-in-premature-infants-and-asthma-development

\section{Hosted file}

table 3.docx available at https://authorea.com/users/330315/articles/457134-the-associationbetween-early-life-salivary-cytokines-in-premature-infants-and-asthma-development

\section{Hosted file}

table 4.docx available at https://authorea.com/users/330315/articles/457134-the-associationbetween-early-life-salivary-cytokines-in-premature-infants-and-asthma-development

\section{Hosted file}


table 5.docx available at https://authorea.com/users/330315/articles/457134-the-associationbetween-early-life-salivary-cytokines-in-premature-infants-and-asthma-development

(A)

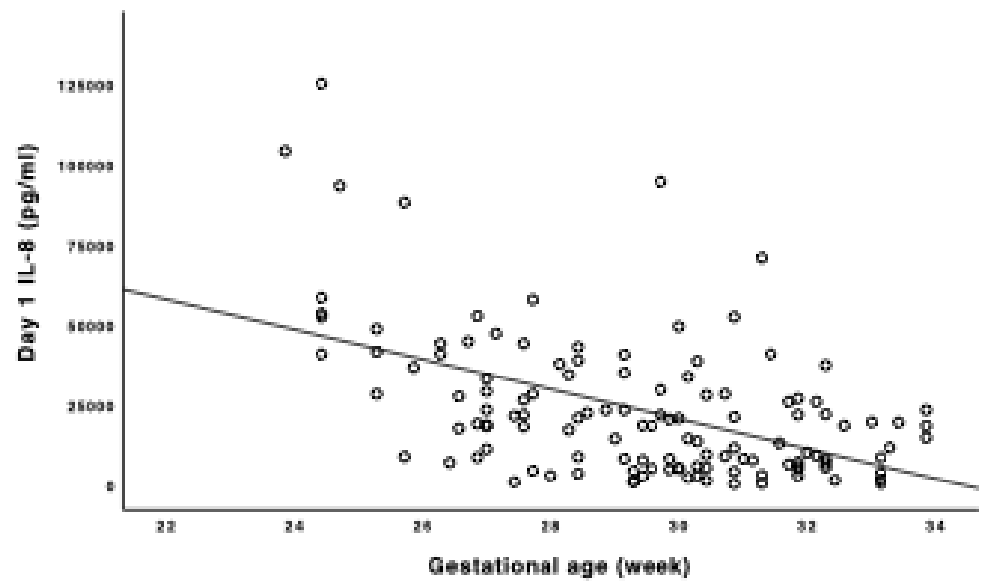

(B)

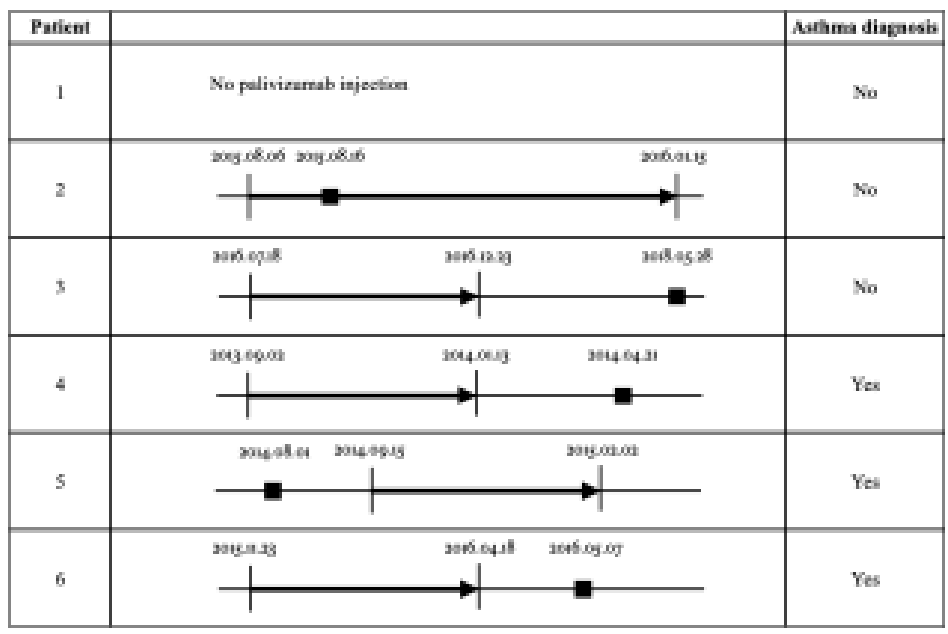

(C)

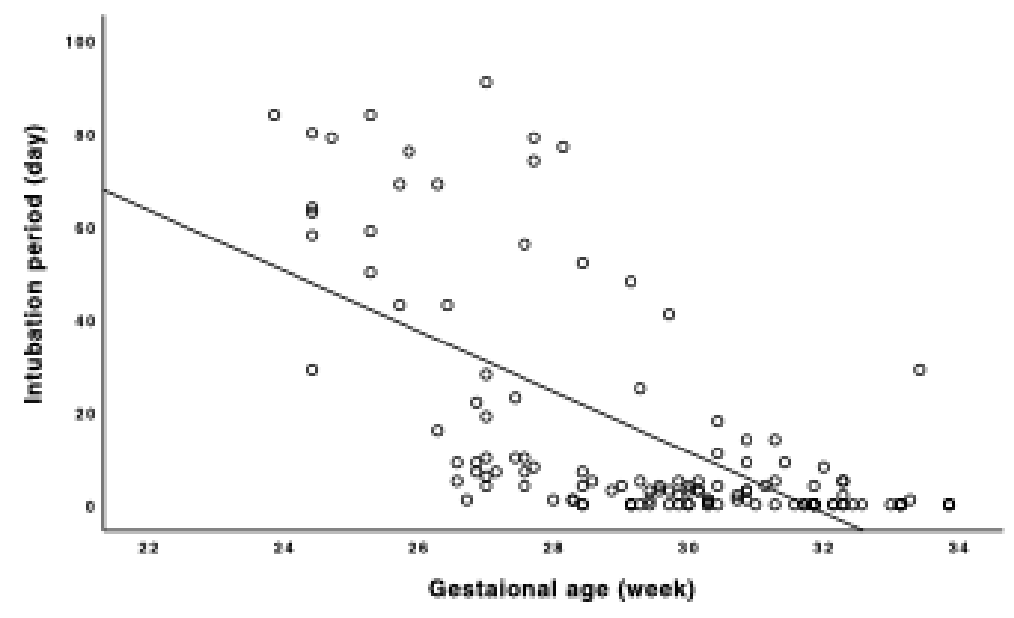

